\title{
Situation de la rédaction professionelle dans l'entreprise: I-Résultats d'une enquête effectuée, en 1992, auprès de 26 entreprises (Sociétés d'état et Sociétés commerciales publiques et privées) situées dans la région montréalaise *
}

\author{
Louise Larivière \\ Université de Montréal et Université Concordia
}

This paper documents the results of a study of language-related tasks in Montreal business settings. Of all language activities undertaken in the language departments and other related services of those businesses surveyed, technical writing is four times less important than translation. An additional finding is that technical writing, in the strictest sense of the term, is done mostly in English, even in French-speaking (Francophone) businesses.

La rédaction occupe près de quatre fois moins de place que la traduction parmi les activités langagières qui s'exercent dans les services linguistiques et autres services qui dispensent ces activités dans certaines entreprises montréalaises. Quant à la rédaction technique, au sens strict du terme, elle s'effectue surtout en anglais même dans certaines entreprises francophones.

*Une première version de cette partie de ce travail a fait l'objet d'une communication lors du Congrès des Sociétés savantes, dans le cadre de l'Association canadienne des professeurs de rédaction technique et scientifique, Charlottetown (PE), mai 1992. Certaines données ont été ajoutées à l'été 92, ce qui modifie les premiers résultats présentés.

La deuxième partie de ce travail: "Situation de la rédaction professionnelle dans l'entreprise: II - Résultats d'une enquête effectuée en 1994 auprès de 20 ministères de la législature du Québec» a fait l'objet d'une communication présentée lors du congrès The International Professional Communication Conference (IPCC) de l'organisme Institute of Electrical and Electronics Engineers Inc. (IEEE), Banff (AB), 28-30 septembre 1994. 


\section{Objet}

LE PRÉSENT ARTICLE ANALYSE les résultats d'une enquête effectuée en 1992 et qui avait pour but d'examiner la place que le milieu des entreprises accordait aux activités langagières, particulièrement à la rédaction professionnelle telle que nous l'avons définie dans une étude antérieure (Larivière, 1986) ${ }^{1}$, et ce, dans une période difficile de récession.

Deux faits sont à l'origine de ces préoccupations. Le premier porte sur l'hypothèse généralement admise, dans les milieux langagiers, que les services linguistiques des entreprises montréalaises consacrent leurs activités davantage à la traduction qu'à la rédaction. Cette affirmation pour se justifier se devait, toutefois, d'être quantifiée.

Le second est relié à la difficulté, depuis les années 80 , de gérer les services linguistiques qui ont dû faire face à des réductions de personnel et des compressions budgétaires. Ces dernières ont eu, bien souvent, pour effet de réduire ces services à une seule personne, de limiter leurs activités ou, tout simplement, d'y mettre fin. Ainsi, à Hydro-Québec, pour citer un exemple, il n'y a plus d'entité administrative distincte pour dispenser les services linguistiques comme autrefois; ces derniers sont donnés en sous-traitance ou assumés par des conseillers et des conseillères en communication répartis dans différentes unités. Aussi a-t-on proposé diverses mesures pour contrer ces difficultés: nécessité de "faire différemment avec moins", d'utiliser la fonction «marketing», de recourir à des ressources externes (Cornibert, 1991), bref de repenser l'état de la profession et de faire face au marché en tenant compte, notamment, de l'informatisation, des nouvelles technologies et $\mathrm{du}$ virage éditique (Verrier, 1991).

Pour savoir quelle était la situation réelle, nous avons alors fait porter notre enquête sur la nature et la proportion de l'ENSEMBLE des activités langagière encore offertes par divers services de certaines entreprises, DONT LES SERVICES LINGUISTIQUES-dans la mesure où ceux-ci étaient évidemment maintenus-et sur la composition de ces services.

Ultimement, nous visions à ce que les données recueillies permettent 1) de faire ressortir la part accordée aux activités de rédaction, particulièrement la rédaction technique, par rapport aux autres activités langagières; 2) de mieux orienter le contenu des cours de rédaction commerciale et de rédaction technique dispensés dans les universités et 3 ) de mieux faire connaître à la population étudiante les débouchés existants en matière d'activités langagières. 


\section{Méthodologie}

Pour ce faire, nous avons procédé par échantillonnage et fait parvenir un questionnaire à divers services ${ }^{2}$ de 40 entreprises de la région de Montréal choisies à partir de répertoires ${ }^{3}$ et en fonction des critères suivants ${ }^{4}: 1$. entreprise canadienne; 2 . avoir son siège social dans la région de Montréal ou de Toronto; 3. compter un personnel de plus de 500 personnes; 4 . oeuvrer dans le domaine technique. Sur les 40 entreprises sollicitées, 26 ont répondu à notre appel ${ }^{5}$. Nous comptabilisons toutefois 29 questionnaires à cause des deux divisions distinctes d'une entreprise ${ }^{6}$ et des trois directions aux structures et aux intérêts diversifiés d'une société d'État'. Le pourcentage de réponses est, donc, de près de $73 \%$. Trois entreprises dérogent à certains critères établis ${ }^{8}$.

Les entreprises répondantes se répartissent en quatre sociétés d'État (deux québécoises et deux fédérales) ${ }^{9}$ ayant toutes leur siège social à Montréal, 21 sociétés commerciales, publiques ou privées, et un cabinet de génie-conseil dont 10 ont leur siège social dans la région torontoise et 12 dans la région montréalaise. Elles oeuvrent dans les secteurs suivants ${ }^{10}$ : électronique/ électrotechnique (4), transports (4), pâtes et papiers (3), énergie (3), informatique/bureautique (2), aéronautique/aérospatiale (2), télécommunications (2), ingénierie (2), sidérurgie (1), automobile (1), produits de beauté (1), services de sécurité et régulation (1) ${ }^{11}$.

\section{Résultats}

Les résultats sont analysés en suivant l'ordre de déroulement du questionnaire qui comportait deux grandes parties. La première touchait à la nature des services offerts soit la traduction, la terminologie, la rédaction, la révision, la formation linguistique et autres services. La seconde portait sur la caractérisation du personnel langagier: sa composition (personnel interne et externe), les critères de son embauche et sa répartition à l'intérieur des unités administratives.

\section{Nature des Services Offerts}

Le Tableau 1 énumère chacune des activités offertes et donne, pour chacune d'elles, le pourcentage des entreprises qui offrent cette activité et le pourcentage que cette activité représente par rapport à l'ensemble des activités offertes. On se rapportera à ce tableau pour toutes les données commentées ${ }^{12}$. 
Tableau 1: Répartition, en pourcentage, des activités offertes

\begin{tabular}{|c|c|c|}
\hline $\begin{array}{c}\text { Nature de l'activité } \\
\text { offerte }\end{array}$ & $\begin{array}{c}\% \text { des } \\
\text { cntreprises } \\
\text { qui offrent } \\
\text { cette } \\
\text { activité }\end{array}$ & $\begin{array}{c}\text { \% que cettc } \\
\text { activité représente } \\
\text { par rapport à } \\
\text { l'enscmble des } \\
\text { activités offertes }\end{array}$ \\
\hline \hline $\begin{array}{c}\text { traduction } \\
\text { français......anglais } \\
\text { anglais....français } \\
\text { autres langues }\end{array}$ & $93 \%$ & $37 \%$ \\
\hline révision & $93 \%$ & $60 \%$ \\
\hline terminologic & $93 \%$ & $3 \%$ \\
\hline formation linguistique & $38 \%$ & $19 \%$ \\
\hline rédaction & $34 \%$ & $9 \%$ \\
\hline autres & $31 \%$ & $14 \%$ \\
\hline
\end{tabular}

\section{a. Traduction}

La majorité des entreprises (27 sur 29) effectuent des travaux de traduction dans une proportion de $93 \%$ pour ce qui est de la traduction du français vers l'anglais et de la traduction de l'anglais vers le français. Toutefois, seulement $45 \%$ des entreprises effectuent de la traduction dans d'autres langues, soit l'italien, le suédois, le tchèque, le chinois mandarin, l'arabe, l'allemand et l'espagnol, ces deux dernières langues étant les plus utilisées.

Par rapport à l'ensemble des activités offertes, les travaux de traduction représentent les pourcentages suivants: $60 \%$ pour la traduction de l'anglais vers le français, $37 \%$ du français vers l'anglais et $3 \%$ dans d'autres langues.

\section{b. Terminologie}

La terminologie, considérée comme une activité connexe à la traduction, est exercée dans $76 \%$ des entreprises, bien que ces dernières n'y consacrent que $9 \%$ de leur temps. Sept entreprises ont mentionné soit ne pas ou ne plus avoir d'activités terminologiques, soit avoir réduit leurs activités pour cause de réorganisation, de «rationalisation» ou de réduction de personnel. Aussi, dans ces dernières, on n'alimente plus les banques de données, ne crée plus de répertoires terminologiques, ni n'effectue plus de recherches ponctuelles 
pour répondre à des consultations téléphoniques.

La liste suivante énumère les diverses activités terminologiques exercées, accompagnées du nombre d'entreprises qui les exercent.

Liste des activitées terminologiques exercées dans les entreprises

- réponses aux consultations téléphoniques sur des problèmes de terminologie (11);

- création et mise à jour de lexiques et de vocabulaires bilingues et multilingues (9);

- recherches ponctuelles (7) [pour satisfaire des besoins de traduction];

- gestion (constitution et alimentation) de banques de données terminologiques d'entreprise informatisées (6);

- normalisation des titres de fonctions et autres termes (6);

- dépouillement de documents divers et de textes traduits (5);

- $\quad$ rédaction et révision de fiches (5);

- recherches thématiques (4);

- maintien d'un centre de documentation et recherches documentaires (3);

- rubrique de terminologie dans le journal d'entreprise (2).

\section{c. Rédaction}

En matière de rédaction, nous avons demandé quelle était la nature des documents rédigés, tant pour répondre aux besoins du service lui-même que pour répondre aux besoins d'autres services, et quel pourcentage ces documents représentaient dans l'ensemble des activités de rédaction.

Nous avons compilé seulement les données des entreprises qui 
déclaraient effectuer de la rédaction originale en français ou de la rédaction à la fois en français et en anglais. Nous avons exclu les données qui portaient sur la rédaction en anglais et l'aide à la rédaction, bien que certaines entreprises ne démarquaient pas toujours clairement les limites entre la rédaction et l'aide à la rédaction. Nous avons également exclu des pourcentages trois entreprises qui effectuaient beaucoup de rédaction en dehors des services linguistiques (génie-conseil et relations publiques) parce que leurs taux de pourcentage très élevés contrastaient trop avec ceux des autres services et faussaient, de ce fait, les données.

Par conséquent, nous établissons à 34\% (soit 10 entreprises sur 29) le pourcentage des entreprises qui s'adonnent à des activités de rédaction, lesquelles censtituent $16 \%$ de l'ensemble des activités langagières. Encore faut-il dire que parmi ces 10 entreprises, il se trouve deux sociétés d'État québécoises soit Sidbec-Dosco et le service de la Communication commerciale d'Hydro-Québec dont le pourcentage des activités de rédaction se chiffre, respectivement, à 15 et $30 \%$.

Par ailleurs, trois entreprises, dont deux ont leur siège social à Montréal, ne font de la rédaction originale qu'en anglais (et cette rédaction n'est que technique). On invoque, pour ce faire, la technologie de pointe dans certains domaines comme l'aéronautique, le marché international, la clientèle qui est surtout de langue anglaise, ainsi que des raisons de temps et de commodité. Une entreprise traduit systématiquement ses manuels techniques en français, tandis qu'une autre le fera à la demande expresse de la clientèle. Trois entreprises répondantes soulignent, en commentaires, la piètre qualité du français écrit et la nécessité d'avoir des cours de rédaction professionnelle. C'est la raison pour laquelle un des répondants juge bon, faute de mieux, de faire "missionnellement» de l'aide à la rédaction.

Le Tableau 2 indique quel pourcentage la rédaction de chacun des documents ADMINISTRATIFS ET TECHNIQUES suivants représente dans l'ensemble des activités de rédaction. 
Tableau 2: Pourcentage des documents administratifs et techniques rédigés

\begin{tabular}{|c|c|c|c|}
\hline & $\begin{array}{l}\text { type de documents } \\
\text { administratifs ou } \\
\text { techniques }\end{array}$ & $\begin{array}{l}\% \text { que ce } \\
\text { document } \\
\text { représente } \\
\text { dans } \\
\text { l'ensemble } \\
\text { des activités } \\
\text { de rédaction }\end{array}$ & $\begin{array}{c}\text { nombre } \\
\text { d'entreprises } \\
\text { (sur 10) } \\
\text { ayant } \\
\text { exprimé un } \\
\%\end{array}$ \\
\hline 1 & lettres & $9 \%$ & 8 \\
\hline 2 & notes de service & $13 \%$ & 7 \\
\hline 3 & comptes rendus & $2 \%$ & 3 \\
\hline 4 & proces-verbaux & $4 \%$ & 3 \\
\hline 5 & communiqués & $9 \%$ & 7 \\
\hline 6 & avis & $7 \%$ & 5 \\
\hline 7 & appels d'offre & $3 \%$ & 2 \\
\hline 8 & offres d'emploi & $2 \%$ & 4 \\
\hline 9 & matćricl publicitaire & $27 \%$ & 5 \\
\hline 10 & rapports annucls & $10 \%$ & 2 \\
\hline 11 & mímoires & $2 \%$ & 1 \\
\hline 12 & Etudes & $9 \%$ & 2 \\
\hline 1.3 & autres rapports & $12 \%$ & 2 \\
\hline 14 & $\begin{array}{l}\text { manucls d'cutreticn } \\
\text { et d'utilization }\end{array}$ & $29 \%$ & 4 \\
\hline 15 & $\begin{array}{l}\text { description de } \\
\text { tâches }\end{array}$ & $1 \%$ & 4 \\
\hline 16 & fiches technigues & $5 \%$ & 4 \\
\hline 17 & directives & $9 \%$ & 3 \\
\hline 18 & modes d'emploi & $5 \%$ & 3 \\
\hline 19 & normes & $2 \%$ & 4 \\
\hline
\end{tabular}


Même si le taux de réponses est peu élevé, il semble que les documents de correspondance (lettres, notes et communiqués), le matériel publicitaire, les rapports ainsi que les manuels techniques soient les documents les plus rédigés.

Nous avons également demandé, dans la section sur la rédaction, s'il y avait d'autres documents qui s'ajoutaient à la liste soumise et s'il existait d'autres types d'activité de rédaction. Étant donné la confusion dans certaines réponses à ces deux questions, nous les avons traitées globalement. Nous indiquons ces ajouts dans la liste suivante.

Liste de documents autres qui font l'objet de rédaction

- journaux d'entreprise et bulletins d'information;

- allocutions pour la haute direction, discours pour diverses occasions et présentations à des fins de marketing ou de subventions gouvernementales;

- chroniques linguistiques, scripts audio-visuels, guides à l'intention du personnel, curriculums, analyses financières, brochures et dépliants de toutes sortes, messages à la clientèle, textes pour les stands d'exposition ainsi que concours linguistiques.

\section{d. Révision}

La révision est une activité reliée tant aux activités de traduction que de rédaction. Elle est exercée dans 93\% des entreprises (soit 27 sur 29) et représente $19 \%$ de l'ensemble des autres activités langagières (soit un écart entre les entreprises qui varie de 2 à $70 \%$ ).

Le degré de révision ou de contrôle de la qualité des textes dépend des personnes à qui ces textes ont été confiés: les documents traités en dehors de l'entreprise font généralement l'objet d'une révision exhaustive ainsi que ceux qui proviennent des services de l'entreprise autres que des services linguistiques; les textes (rédigés ou traduits) par le personnel langagier sont soumis à moins de contrôle. En effet, pour ce qui est de la traduction à l'intérieur de l'entrprise, on tend de plus en plus vers le contrôle de la qualité plutôt que vers la révision systématique (v. sur cette question Boutry et Contant, 1991), bien que l'interrévision s'exerce toujours. 
Le degré de révision dépend aussi de la nature des textes mêmes: les textes officiels, de prestige, de grande visibilité ou de diffusion (rapports annuels, allocutions par la haute direction) seront davantage révisés. La liste suivante énumère les activités de révision exercées.

\section{Liste des activités de révision}

- conseils et aide à la rédaction de rapports pour le gouvernement ou autres textes à caractère stratégique;

- correction des textes rédigés en français par les divers services des entreprises: manuels, codes, consignes, documents contractuels, brochures, dépliants, communications, articles, scénarios, lettres destinées à la clientèle, notes de service, procès-verbaux, rapports, directives, journaux d'entreprise, devis et spécifications, rapports annuels;

- correction des textes rédigés par des firmes externes de communication;

- correction des traductions confiées à des firmes externes

- interrévision par le personnel langagier interne;

- relecture ct correction d'épreuves.

\section{e. Formation linguistique}

Par formation linguistique, nous entendons tout cours de langue dispensé par l'entreprise, soit des cours d'apprentissage d'une langue seconde, soit des cours de perfectionnement dans sa langue maternelle tel, par exemple, un cours de rédaction de rapports. Il y a $38 \%$ des entreprises qui fournissent une telle formation (soit 11 sur 29). Cette activité représente $14 \%$ de l'ensemble des activités langagières.

Cette formation linguistique peut se donner soit dans les services linguistiques, soit dans d'autres unités administratives telles Ressources humaines, Planification et Développement, Dotation et Développement (4). De plus, lorsque l'entreprise ne dispense pas elle-même la formation, elle 
peut consentir à rembourser le personnel qui va suivre des cours à l'extérieur de l'entreprise, dans la mesure où ces cours sont reliés à l'exercice de ses fonctions (3).

On trouvera dans la liste suivante les activités de formation dispensées au sein des 11 entreprises répondantes, accompagnées du nombre d'entreprises qui les dispensent.

Liste des activités de formation dispensées dans les entreprises

- cours de communications écrites: rédaction de rapports (1), rédaction technique (1), méthodes de rédaction et correction grammaticale (1);

- cours de langues secondes, tant d'anglais que de français (8);

- procédés de perfectionnement divers tels des chroniques dans les journaux d'entreprise (1), des bulletins linguistiques et des guides d'aide à la rédaction destinés au personnel (2), les corrections des textes révisés (1), des concours organisés à l'intention du personnel salarié et syndiqué (1), des manuels de consultation mis à la disposition du personncl (1);

- présentations faites aux pigistes et à la clientèle afin de leur préciser la nature du travail linguistique effectué par l'entreprise, de les sensibiliser à ses exigences en matière linguistique et de leur faire connaître les outils qu'clle utilise et l'aide que ces derniers pourraient leur apporter (1).

\section{f. Autres activités langagières}

Les activités résiduelles, sous le titre de «autres activités langagières" sont exercées dans $31 \%$ des entreprises (9 sur 29). Ces activités, qui représentent $5 \%$ de l'ensemble des activités langagières, sont les suivantes:

\section{Autres activitées langagières}

- services d'aide à la rédaction à l'intention d'autres services (1); 
- réponses à des consultations verbales sur des questions de grammaire et de style (5);

- concours et jeux linguistiques (dictées, mots croisés) pour le personnel lors de la Semaine de français (1);

- rubrique de langue (grammaire, style) dans le journal d'entreprise (1);

- $\quad$ services de publication (éditique), de graphisme et de soutien à la programmation (réseaux, bases de données) (1);

- établissement et diffusion de normes propres aux services linguistiques (1);

- recherches documentaires (3);

- $\quad$ relecture et correction d'épreuves (2);

- cours de rédaction technique (1).

Il est à noter que les concours et les rubriques de langue dans les journaux d'entreprise ont été considérés par d'autres entreprises comme étant des activités de formation et apparaissent sous cette rubrique. Les recherches documentaires sont rattachées pour d'autres entreprises aux activités de terminologie. Par ailleurs, la relecture et la correction d'épreuves auraient pu appartenir à la rubrique révision. Quant aux cours de rédaction technique, ils auraient davantage leur place en formation linguistique. Si l'on considère la réponse fournie dans la section "formation linguistique" par l'entreprise qui a classé la rédaction technique parmi les autres activités, on s'aperçoit que la formation linguistique a été comprise par cette entreprise comme étant des cours d'apprentissage d'une langue seconde. Comme, pour cette entreprise, ces cours se donnent en dehors des services linguistiques, on a tout simplement mentionné le fait et inclus les cours de rédaction technique comme étant une activité autre. Toutes ces variations dans la répartition des activités langagières ne sont, en définitive, que question de points de vue ou d'organisation interne. 


\section{Caractérisation du personnel langagier}

\section{a. Composition du personnel langagier}

À la question: «À qui votre entreprise fait-elle appel pour offrir des services linguistiques: au personnel langagier interne, au personnel langagier externe, ou aux deux et dans quelle proportion?», certaines entreprises ont répondu en établissant des pourcentages par activité linguistique. Le taux de réponse pour cette question est de $97 \%$ ( 28 sur 29 ). Sur les 28 répondantes, trois entreprises n'ont pas indiqué de pourcentage.

Le Tableau 3 schématise la composition du personnel langagier.

Tableau 3: Composition du personnel langagier

\begin{tabular}{|c|c|c|}
\hline lype de persomnel & $\begin{array}{l}\text { réponses en \% par } \\
\text { nombres d'entreprises }\end{array}$ & $\begin{array}{c}\text { \% de réponses } \\
\text { sur l'ensemble } \\
\text { des } \\
\text { entreprises }\end{array}$ \\
\hline personnel interne & $100 \%$ (1) terminologic & $3 \%$ \\
\hline personnel externe & $\begin{array}{l}100 \%(2) \text { traduction } \\
100 \% \text { (1) formation }\end{array}$ & $10 \%$ \\
\hline $\begin{array}{c}\text { personnel } \\
\text { interne/externe } \\
\text { sans pourcentage } \\
\text { avec pourcentage } \\
\text { interne = externe } \\
\text { interne } \neq \text { externe } \\
\text { interne (bureau régional) } \\
\text { externe (siege social) }\end{array}$ & $\begin{array}{c}28 \operatorname{sur} 29 \\
03 \text { sur } 28 \\
25 \operatorname{sur} 28 \\
=50 \%(5) \\
-50 \%(7) /-50 \%(7) \\
+50 \%(12) /+50 \%(12) \\
100 \%(1) \\
60 \%(1)\end{array}$ & $\begin{array}{c}97 \% \\
11 \% \\
89 \% \\
20 \% \\
76 \% \quad 28 \% \\
48 \% \\
4 \%\end{array}$ \\
\hline
\end{tabular}


Une entreprise précise qu'elle utilise uniquement du personnel interne $(100 \%)$ pour ses travaux de terminologie, ce qui représente $3 \%$ de l'ensemble des entreprises. Deux autres notent qu'elles utilisent uniquement du personnel externe $(100 \%)$ pour ce qui est de la traduction et une autre uniquement du personnel externe $(100 \%)$ pour ce qui est de la formation; ces trois entreprises représentent $10 \%$ de l'ensemble des répondants. Il y a 28 répondants qui utilisent à la fois du personnel externe et interne (97\%) dont trois n'ont pas établi de proportion (11\%). Sur les 25 autres (89\%) qui ont établi des pourcentages, cinq (20\%) en utilisent dans la même proportion (50-50) dont l'un uniquement dans le domaine de la traduction; 19 (76\%) dans des proportions différentes: sept (moins de 50\%) et 12 (plus de 50\%,) en ce qui concerne le personnel interne, soit 28 et $52 \%$ de l'ensemble, et, inversement 12 (moins de $50 \%$ ) et sept (plus de $50 \%$ ) pour le personnel externe, soit 52 et $28 \%$ de l'ensemble, dont l'un uniquement pour ce qui est de la rédaction; un (4\%) qui établit ses proportions en fonction des lieux de travail: $100 \%$ de personnel interne au bureau régional et $60 \%$ de personnel externe au siège social.

\section{b. Caractéristiques $d u$ personnel externe}

Les travaux de sous-traitance effectués à l'extérieur des entreprises sont confiés à des agences, à des cabinets, à des pigistes ou à des indépendants dans les proportions qui apparaissent au Tableau 4.

Tableau 4: Répartition du personnel de sous-traitance

\begin{tabular}{|c|c|c|}
\hline Types de sous-traitance & $\%$ & Nombre d'entreprises \\
\hline \hline agences & $32,4 \%$ & 05 \\
\hline cabinets & $50,3 \%$ & 14 \\
\hline pigistes et indépendants & $69,5 \%$ & 22 \\
\hline
\end{tabular}


Le personnel externe est choisi en fonction des critères suivants:

\section{Qualifications exigées du personnel langagier externe}

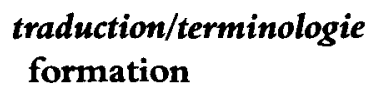

- $\quad$ formation universitaire (2)

- formation en rédaction technique (1)

accréditation

- $\quad$ accréditation profesionnelle (STQ, ATIO, etc.) (1)

\section{expérience}

- expérience pertinente/dans le domaine spécifique à l'entreprise (9)

- $\quad$ expérience antéricure acquise dans l'entrcprisc (4)

- $\quad \mathrm{CV}+$ exemples de travaux déjà réalisés (1)

- $\quad$ pas de débutants (1)

\section{compétences}

- $\quad$ travaux de grande qualité (6)

- compétences linguistiques/nivcaux de langue/qualité du français ou de l'anglais (4)

- réussite aux tests de traduction (sous forme de petit contrat de travail ou autrement) (2)

- consultation des banques de terminologie et autres sources (1)

- $\quad$ minimum de révision (1) 


\section{connaissances}

- $\quad$ spécialisation/connaissance du sujet ou du domaine traité (9)

- connaissance de l'entreprise (5)

- connaissance de WordPerfect 5.1 ou autres logiciels (2)

\section{réputation}

- références ou recommandations faites par des spécialistes (3)

- cabinet reconnu (2)

- relations professionnelles déjà établies avec les membres de la direction (1)

- pas d'inconnus (1)

\section{qualités professionnelles}

- $\quad$ respect des ćchéances (6)

- $\quad$ rapidité (6)

- grande disponibilité (au besoin venir travailler sur place) (4)

- $\quad$ efficacitć/cxpertise (3)

- capacité de communiquer avec la clientèle (2)

- capacité d'utiliser la documentation maison (1)

- capacité d'aller chercher les renseignements nécessaires (1)

- $\quad$ service courtois et personnalisé (1) 
- $\quad$ rigueur technique (1)

- professionnalisme (1)

qualités personnelles

- $\quad$ autonomie (2)

- flabilité (2)

- $\quad$ souplesse (1)

- capacité de s'adapter aux exigences propres à l'entreprise (1)

\section{tarification}

- $\quad$ tarifs concurrentiels (8)

\section{équipement informatique (matériel et logiciel)}

- $\quad$ ordinateur équipé de modem/télécopicur (4)

- $\quad$ type de logiciel utilisé (1)

- $\quad$ équipement compatible avec celui de l'entrcprise (1)

\section{formation}

- connaissance des méthodes d'enseignement utilisées dans l'entreprise

- $\quad$ possibilité de s'adapter à des horaires variés

\section{relations publiques}

- compétence dans les relations publiques/relations industriclles 


\section{c. Caractéristiques du personnel interne}

Pour ce qui est du personnel langagier interne, nous voulions savoir à quelle(s) unité(s) administrative(s) il était rattaché et de quel type de personnel étaient composées ces unités. Ce personnel fait partie de diverses unités administratives dont certaines, malgré diverses appellations, s'occupent uniquement de questions linguistiques: services linguistiques, centre de traduction, services français, communications françaises, service de francisation, traduction, service de la traduction et de la rédaction technique, section traduction, service/direction des communications. Ces unités s'occupent principalement de traduction et peuvent aussi offrir des services de terminologie selon le personnel en place. Certaines unités ne s'occupent que de rédaction: communication commerciale et équipe journaux d'entreprise d'Hydro-Québec. Dans certaines entreprises la traduction relève des services linguistiques/traduction tandis que la rédaction relève des relations/ affaires publiques. Dans d'autres, la traduction relève de services divers: affaires ou relations publiques, secrétariat et services juridiques. Il est, cependant, un fait certain: il se fait plus de rédaction dans les services d'affaires ou de relations publiques que dans les services linguistiques. L'Annexe I fournit tous les renseignements quant à la composition du personnel interne pour chacune des entreprises.

Pour ce qui est des critères d'embauche du personnel langagier interne, on exige les qualifications suivantes:

\section{Qualifications exigées du personnel langagier interne}

\section{traduction/terminologie formation}

- $\quad$ B.A. (8), diplôme (7) ou maîtrise (3) en traduction ou en terminologie selon le cas

- formation universitaire (5)

\section{accréditation}

- $\quad$ membre agréé de la STQ (1)

- membre de la Corporation des traducteurs et interprètes (1) professionnnels du Québec (1) 
expérience

- solide expérience de travail/expérience pertinente de plusieurs années en traduction (11)

- trois à cinq ans d'expérience dans le domaine/expérience minimale de trois ans pour traducteurs et rédacteurs (2)

- $\quad$ minimum cinq ans d'expérience (superviseuse) (1)

- $\quad$ expérience en terminologie (2)

\section{compétence}

- $\quad$ travaux de qualité (1)

- $\quad$ aptitudes pour les domaines techniques (1)

\section{connaissances}

- connaissance approfondie du français et de l'anglais/version et thèmc: anglais ct français (4)

- bonne connaissance du traitement de textes (1)

\section{réputation}

\section{qualités professionnelles}

- $\quad$ grande curiosité encyclopédique (1)

- $\quad$ grande disponibilitć (1)

- grande capacité de travail même sans révision (1)

- $\quad$ autonomie (1)

- $\quad$ rapidité (1)

- polyvalence (1) 
- capacité de traduire ou de travailler dans des délais impartis (1)

- $\quad$ souci du travail soigné (1)

- $\quad$ aptitude au travail en équipe (1)

\section{qualités personnelles}

- $\quad$ entregent/bon contact avec les gens (2)

- $\quad$ souplesse dans les relations humaines (3)

- $\quad$ tact et diplomatic (1)

- $\quad$ ouverture d'esprit (1)

\section{domaines divers}

\section{enseignement du français}

- $\quad$ B.A. en lettres (1)

- diplôme d'enseignement en langue seconde (1)

- $\quad$ plusieurs années d'expérience (1)

\section{communications/relations publiques}

- diplôme de premier cycle en communications, journalisme ou autre domaine connexc (2)

- connaissance du milicu: communications et mćdias d'information (1)

- connaissance du français et de l'anglais (1)

\section{génie-conseil}

- formation universitaire (marketing, communication, journalisme) (1) 
- minimum cinq années d'expérience (secteurs pertinents)

(1)

- bonne connaissance du français (1)

Finalement, nous avons demandé si le personnel embauché avait reçu une formation universitaire qui l'avait suffisamment préparé au marché du travail.

On a jugé la formation universitaire reçue pas toujours satisfaisante bien qu'on lui reconnaisse certains mérites. La liste suivante énumère les réponses négatives et positives données par les entreprises ainsi que le nombre d'occurrences pour chacune d'elles.

Appréciation de la formation universitaire rȩ̧ue par le personnel embauchée

\section{réponses négatives}

- méconnaissance du milieu de l'entreprise privée et pas de préparation aux exigences de ce milieu telles la productivité, la rapidité, le service à la clientc̀le (7)

- manque de sens pratique, de méthode de travail, pas l'habitude de la consultation: personnes-ressources et documentation, manque de précision et de fidélité aux textes (5)

- pas assez d'importance accordée au respect des échéances, au délai d'exécution, pas suffisamment de situations d'écriture ou de traduction sous pression avec des échéances trc̀s courtcs (5)

- trop d'importance accordée à la langue: pureté linguistique, croisade pour la défense de la Langue française et pas assez à la réalité du monde linguistique des entreprises et à sa nécessité de vulgariser et de simplifier davantage (4)

- faiblesses en matière de syntaxe et d'orthographe et manque de culture générale (3) 


\section{réponses positives}

- la formation universitaire sert de base, permet d'acquérir une certaine maturité, une rigueur intellectuelle et de mieux comprendre le processus global de la traduction, mais il y a lieu de s'adapter à la réalité de chaque entreprise et de compléter par une formation sur le tas et une connaissance du domaine qui est indispensable (7)

- oui, mais c'est l'expérience qui sert de critère de sélection (2)

- $\quad$ oui (3), mais préparation en termes de qualité du travail seulement pas pour les connaissances techniques (2)

\section{Conclusions}

De ces résultats, nous pouvons tirer les conclusions suivantes :

\section{Nature des services offerts}

Conformément à l'hypothèse posée au départ, la traduction occupe la plus large part dans les activités langagières. La majorité des entreprises $(60 \%)$ exerce cette activité, principalement de l'anglais vers le français. Avec l'avènement de la mondialisation des marchés, il sera intéressant de suivre l'évolution des troisièmes langues.

La terminologie qui a déjà occupé une place importante ne constitue plus que $9 \%$ des activités langagières. Étant une activité coûteuse (en temps et en salaire), elle est probablement celle qui souffre le plus des compressions budgétaires.

Comme il fallait s'y attendre, également, l'analyse démontre que la rédaction occupe peu de place, soit $16 \%$ de toutes les activités langagières, ce qui représente presque quatre fois moins de place que la traduction. On ne peut pas, cependant, conclure que l'on écrit peu en français dans les entreprises québécoises puisque ce ne sont pas tous les services des entreprises qui ont été soumis au questionnaire. Une étude à l'échelle de chacune de toutes les entreprises serait, sans doute, plus concluante, mais constituerait une tâche longue, coûteuse et probablement impraticable.

Ce que l'on peut affirmer, cependant, c'est que la rédaction technique, dans certaines entreprises de haute technologie, s'effectue en anglais même 
dans celles qui ont une direction francophone et dont le siège social se situe dans la région métropolitaine. Lorsque ces entreprises affichent, dans les journaux, des postes à combler en rédaction technique, c'est pour y rédiger en anglais, qu'elles le mentionnent ou non. Il y a donc peu de place sur le marché du travail pour des rédacteurs et des rédactrices qui veulent effectuer de la rédaction technique en français.

La révision, quant à elle, occupe une place plus ou moins importante selon les entreprises pour une moyenne de $19 \%$ sur l'ensemble des activités langagières. Ce sont les textes officiels, de prestige, de grande visibilité ou de diffusion qui sont surtout révisés de même que ceux du personnel des firmes externes. À l'intérieur de l'entreprise, on tend de plus en plus vers le contrôle de la qualité.

Parmi les activités de formation linguistique, les cours de langues secondes (que ce soit l'anglais ou le français) prédominent sur toutes les autres. Cette activité qui peut s'exercer dans les services linguistiques ou dans d'autres unités administratives compte pour $14 \%$ dans l'ensemble des activités langagières.

D'autres activités linguistiques de nature diverse s'exercent dans une proportion de $5 \%$. Les réponses aux consultations verbales sur des questions de grammaire ou de style sont le plus souvent mentionnées comme activité résiduelle.

\section{Caractérisation du personnel langagier}

Dans une proportion de $97 \%$, les entreprises utilisent à la fois du personnel langagier interne et externe, parfois dans une proportion égale (5050). Par ailleurs, il y a autant d'entreprises (48\%) qui emploient moins de $\mathbf{5 0} \%$ de personnel interne que d'entreprises qui emploient plus de $50 \%$ de personnel externe; inversement, il y a autant d'entreprises (52\% ) qui emploient plus de $50 \%$ de personnel interne que d'entreprises qui emploient moins de $50 \%$ de personnel externe.

Pour ce qui est du personnel externe, les entreprises font affaire majoritairement avec des pigistes et indépendants, dans $70 \%$ des cas, qu'elles embauchent en fonction des critères suivants: expérience, qualité des travaux, connaissance du domaine de l'entreprise, respect des échéances, tarifs concurrentiels et possession d'équipement informatique. Quant au personnel interne, on mise surtout sur la formation universitaire, l'expérience de travail, les connaissances linguistiques, l'entregent et la souplesse dans les relations humaines. Comme l'expérience compte pour beaucoup dans 
l'embauche du personnel, qu'il soit externe ou interne, les grandes entreprises excluent, de ce fait, les nouveaux et les nouvelles diplômés qui voudraient entrer sur le marché du travail.

Par ailleurs, les entreprises semblent plutôt insatisfaites de la formation universitaire reçue par leur personnel langagier. On lui reproche surtout de ne pas préparer suffisamment la population étudiante aux exigences du milieu de travail, d'accorder trop d'importance à la «pureté linguistique», de ne pas inculquer de bonnes habitudes de travail et de ne pas assez insister sur la culture générale. Elles lui reconnaissent, toutefois, le mérite d'apporter une certaine maturité et une rigueur intellectuelle, d'enseigner les principes de la traduction et d'inculquer des connaissances sur la langue. En définitive, cependant, c'est la formation sur le tas qui est la plus bénéfique pour s'initier au domaine de travail.

Somme toute, cette étude, qui se voulait avant tout EXPLORATOIRE, aura permis de confirmer ce que l'on savait par hypothèse, à savoir que la traduction prime sur la rédaction originale en français dans les services linguistiques des grandes entreprises montréalaises et que la rédaction technique s'effectue d'abord en anglais. En d'autres termes, il existe, dans ce pays aux "deux solitudes", une «solitude» qui rédige et une autre qui traduit. Par conséquent, les personnes intéressées à la rédaction en français auraient avantage à s'orienter du côté des communications et des relations publiques. De plus, cette étude souligne, à nouveau, la triste réalité qui fait que les services linguistiques ont dû réduire ou abandonner leurs effectifs et leurs activités pour se plier aux exigences d'une période de récession difficile. Ces services seront peut-être amenés à se redéfinir à l'avenir. 


\section{Annexe 1}

\section{Répartition du personnel interne}

\section{Unités administratives / Nombres de personnes / Titre}

\section{Canadien National}

Services linguistiques

1 directrice de réseau

1 secrétaire

3 directrices

traduction

terminologie

enseignement langues

1 chef de bureau

3 coordonnatrices

trad. administrative

trad. technique

trad. anglaise

1 gestionnaire de réseau

7 traducteurs

3 administratifs

3 techniques

1 anglais

2 préposées au trait. de textes

4 terminologues

1 documentaliste

1 adj.-bureautique intégrée

1 commis (enseignement)

7 professeurs

1 conseiller pédagogique

\begin{tabular}{|c|c|c|}
\hline \multicolumn{3}{|l|}{ Northern Telecom } \\
\hline Services linguistiques & 22 & 1 directeur \\
\hline & & 4 chefs de projet \\
\hline & & 9 traducteurs \\
\hline & & 2 terminologues \\
\hline & & 2 éditique \\
\hline & & 4 soutien administratif \\
\hline
\end{tabular}


IBM Canada Ltée

Services linguistiques

22

directrice, traduction/termino.

3 terminologues

5 traducteurs

5 planificatrices du soutien

français des produits

3 éditique

2 soutien technique

1 directrice planification

1 responsable soutien administratif et financier

\section{Air Canada}

Services linguistiques

12

1 chef de service

3 traducteurs principaux/

réviseurs

5 traducteurs

1 documentaliste

2 employés de soutien

\section{CP Rail}

Centre de traduction

12
6 traducteurs ang.-fran.(incluant réviseurs et directeur)
2 traducteurs fran.-ang.
3 terminologues
1 documentaliste

\section{Hydro-Québec}

Communication commerciale 12

12 conseillers en communication

\section{Hydro-Québec}

Équipe journaux d'entreprise

11

2 conseillers-rédacteurs

en chef

6 journalistes

1 commis

1 secrétaire de rédaction

1 chargée d'équipe

- journaux d'entreprise 
Compagnie pétrolière

Impériale Ltée

9

1 chef des services ling.

Services français

1 terminologue-réviseuse

1 traducteur de syst. inf.

2 conseillers en adaptation

2 traducteurs-réviseurs

2 techniciennes bureautique

Teleglobe Canada Inc.

Services linguistiques

9

6 traducteurs

2 secrétaires

1 gestionnaire

Avon Canada Ltée

Communications françaises

9

Secteur administratif

2

Secteur créatif

7

2 traductrices

1 trad.-rédactrice principale

3 traductrices-rédactrices

1 superviseuse

1 chef de service

1 secrétaire

\section{Bombardier Inc.-Canadair}

Services linguistiques

8

1 superviseur

1 secrétaire

4 traducteurs

1 terminologue

1 professeur de français

\section{Xerox}

Service de francisation

8

1 chef de service

1 terminologue

2 réviseurs

1 coordonnateur adaptation

technique des produits au

marché francophone

1 coordonnateur traduction

automatisée

1 commis

1 spécialiste mise en page 
Le Groupe SNC Inc.

Services de l'information

Service linguistique

7

Affaires publiques

5 traducteurs

2 rédacteurs

\section{Ford du Canada Ltée}

Siège social, Oakville (ON)

4

1 directeur, services français

1 traducteur

2 secrétaires

Bureau régional de l'Est

1

1 traducteur et

coordonnateur des

services administratifs

\section{Dessau inc.}

Marketing, vente et

commmunication

41 vice-président

1 directeur

1 adjointe marketing

1 secrétaire

\section{Compagnie Marconi}

\section{Canada}

Ressources humaines

Relations publiques

4

31 directeur

1 agent des communications

1 secrétaire

Traduction

11 administrateur, francisation et traduction

Via Rail Canada Inc.

Service Équité en matière

3

2 traductrices-réviseuses

d'emploi et langues officielles

Section Traduction

1 terminologue

\section{Spar Aérospatiale Ltée}

Affaires publiques 
Honeywell Limitée

(Département des services de 2 protection)

Service de la traduction et

1

de la rédaction technique

(Régulation)

Direction Communications

1 traductrice et rédactrice technique

1

1 coordonnatrice des services linguistiques

\section{CAE Électronique Ltée}

Resources humaines
1 secrétaire-traductrice

?administrateurs/

administratrices

\section{Stone-Consolidated Inc.}

Service de la traduction

1

1 traductrice

\section{Sidbec-Dosco Inc.}

Service des communications

1

1 traductrice-réviseuse

GE Canada inc.

Communications/

Relations publiques

1

1 coordonnateur - Services

linguistiques

\section{Gaz Métropolitain}

Affaires publiques

1
1 conseiller Affaires
publiques

\section{Domtar}

Secrétariat et services

1 traductrice

juridiques

Zénith Radio Canada Ltée 11 traductrice-réviseuse

\section{Kruger inc.}

Service des communications

(données non disponibles) 


\section{Notes}

1. Nous avons défini la rédaction professionnelle comme «l'ensemble des activités rédactionnelles qui s'exercent dans le milieu du travail» et nous en avons fait une composante de la rédaction spécialisée, au même titre que la rédaction journalistique et la rédaction scientifique. La rédaction spécialisée, connue en anglais sous le vocable de «technical writing", pourrait se traduire par rédaction technique au SENS LARGE; nous n'avons pas retenu, toutefois, cette appellation parce qu'elle est rarement utilisée en français et qu'elle porte à confusion. La rédaction professionnelle se subdivise, à son tour, en divers types de rédaction selon qu'on envisage l'organisme émetteur: rédaction administrative (pour les administrations), rédaction commerciale et rédaction d'affaires (pour les entreprises), ou que l'on envisage le domaine d'application: rédaction de gestion (pour la fonction administrative de l'entreprise) et rédaction technique au SENS STRICT (pour la fonction technique de l'entreprise, soit la fonction reliée à la production. Dans cette étude, il s'agit bien de rédaction professionnelle dans toutes ses composantes, y compris la rédaction technique au sens strict du terme.

2. Nous envisagions, au départ, de tenir compte de tous les services des entreprises que nous avions ciblées. Les premiers contacts téléphoniques avec des chefs de service dans lesquels s'effectuaient des activités langagières ont tôt fait de nous faire voir l'ampleur et la complexité d'une telle recherche pour les moyens limités dont nous disposions. Aussi avons-nous décidé de faire appel aux services linguistiques qui étaient les services les plus aptes à exercer des activités langagières, y compris la rédaction. Dans les entreprises où il n'y avait pas de services linguistiques, nous avons été dirigés vers d'autres services qui s'adonnaient à des activités langagières. Des 29 questionnaires reçus, 19 proviennent de services linguistiques et 10 de services autres dont des services de relations publiques (v. ANNEXE vI). Par conséquent, nous croyons justifié de maintenir le titre de notre étude qui délimite le secteur de notre enquête, soit l'entreprise, à l'exclusion d'autres secteurs comme, par exemple, les maisons d'enseignement. 
À l'intention du public lecteur de Technostyle, non familier avec la réalité des "services linguistiques" tels que nous les connaissons au Québec, nous apportons la précision suivante. Le terme de services linguistiques peut signifier deux réalités différentes: l'une consiste en des entités administratives ("départements") au sein des entreprises, l'autre en des activités langagières ("language activities") exercées à l'intérieur de ces entités. Nous utilisons le terme de services linguistiques dans le PREMIER SENS. Ces services linguistiques ont été créés afin de servir, surtout en français, le personnel et la clientèle des entreprises dont la langue de travail, même au Québec, était majoritairement l'anglais avant la promulgation de la Loi 101 (Charte de la langue française) en 1977. Bien que ces services existassent, dans certaines entreprises, avant l'avènement de cette loi, plusieurs d'entre eux ont été créés pour satisfaire aux exigences de francisation de cette dernière. Aussi offrent-ils, selon leurs taille, leurs revenus ou leurs besoins, des activités langagières diverses afin de diffuser textes et documents en français (et même dans d'autres langues). Ces principales activités sont la traduction, la rédaction ou l'aide à la rédaction, les recherches terminologiques, la révision et les cours de langues.

3. Le Répertoire des services linguistiques d'entreprises et d'organismes de Bell Canada et le Répertoire des membres de l'Association des conseils en gestion linguistique (ACGL). Nous tenons à remercier Nada Kerpan de Bell Canada ainsi que Geneviève Cornibert et Francine Létourneau, respectivement présidente et trésorière de l'ACGL, de nous avoir fourni ces répertoires. Nous remercions, également, Gilles Bélanger, professeur au département de linguistique et de traduction de l'Université de Montréal, pour la documentation qu'il nous a fournie.

4. Nous avons choisi les grandes entreprises parce que ce sont dans ce type d'entreprises qu'il risque de s'y exercer des activités langagières. Nous savions, après avoir fait certaines démarches exploratoires, que peu de ces entreprises s'adonnaient à de la rédaction en français même certaines entreprises francophones C'est ce que nous voulions prouver par des données quantifiées. Par ailleurs, notre enquête aurait pu démontrer 
que ces entreprises, dont plusieurs sont des multinationales, exercent des activités de rédaction parallèle en ce sens qu'elles ne font pas que traduire de l'anglais mais qu'elles rédigent en français parallèlement à l'anglais. Mais ce n'est pas le cas.

5. Nous remercions les entreprises et les personnes responsables dans chacune d'elles qui ont bien voulu répondre à notre questionnaire: Air Canada (Michelle Perreault-Ieraci), Avon Canada Inc. (Lise Bernier-Virgilio), Bombardier Inc.Canadair (Marilena Cortoni), CAE Électronique Ltée (Louise Mercier), Canadien National (Nycole Bélanger), Compagnie Marconi Canada (Jacques Larivière), Compagnie pétrolière Impériale Ltée (Christine Oneson), CP Rail (Jacques Dermine), Dessau inc. (Pierre Lévesque), Domtar (Hélène Ducharme), Ford du Canada Ltée (JeanLéon Bruyère), Gaz Métropolitain (Pierre Durand), Générale électrique du Canada inc. (Richard Doucet), Le Groupe SNC (Christine Christophory), Honeywell Limitée (Carmen Boyer Carignan, Jocelyne Beaupré), Hydro-Québec (Renée Arsenault, Claire Beauchemin, Francine Charest, Marguerite Draper, Françoise Lafontaine, Jean-Marc Lambert), IBM Canada Ltée (Chantal Delorme), Kruger inc. (Louise Vaskelis), Northern Telecom (Danielle Lessard), SidbecDosco Inc. (Marie Crête), Spar Aérospatiale Ltée (Nadine Cressaty), Stone-Consolidated Inc. (Claire Mottard), Teleglobe Canada inc. (Alain Morrissette), Via Rail Canada Inc. (Colette Biche), Xerox Canada Ltée (Paul Michel, Anna Mormina), Zénith Radio Canada Ltée (Christine Lambert).

6. Honeywell Limitée: Régulation et Département des services de protection.

7. Hydro-Québec: Communications Exploitation régionale, Communications Siège social et Équipe Journaux d'entreprise, Communication commerciale.

8. Une entreprise a un personnel de moins de 500 personnes (la firme de génie-conseil Dessau inc.); une autre n'a pas de secteur technique (Avon Canada Inc.); une dernière n'ayant pas de services linguistiques (Gaz Métropolitain), nous avons tenu compte de son service des Affaires publiques et gouvernementales dans lequel il se faisait de la rédaction. Ces 
trois entreprises apportent des considérations diverses qui nous permettent d'effectuer certaines comparaisons.

9. Les sociétés d'État fédérales sont Canadien National et Via Rail Canada Inc. et les québécoises sont Hydro-Québec et SidbecDosco.

10. Les chiffres entre parenthèses, à la suite de certaines données, indiquent le nombre d'entreprises.

11. électronique/électrotechnique (4): CAE Électronique Ltée, Compagnie Marconi Canada, Générale électrique du Canada inc., Zénith Radio Canada Ltée; transports (4): Air Canada, Canadien National, CP Rail, Via Rail Canada Inc; pâtes et papiers (3): Domtar, Kruger inc., Stone-Consolidated Inc; énergie (3): Compagnie pétrolière Impériale ltée, Gaz Métropolitain, Hydro-Québec; informatique/bureautique (2): IBM Canada Ltée, Xerox Canada Ltée; aéronautique/ aérospatiale (2): Bombardier Inc-Canadair, Spar Aérospatiale Ltée; télécommunications (2): Northern Telecom, Teleglobe Canada inc.; ingénierie (2): Dessau inc., Le Groupe SNC; sidérurgie (1): Sidbec-Dosco Inc.; automobile (1): Ford du Canada Ltée; produits de beauté (1): Avon Canada Inc.; services de protection et régulation (1): Honeywell Ltée.

12. Ces pourcentages ont été compilés sur le nombre d'entreprises qui ont inscrit un certain pourcentage et non sur l'ensemble des entreprises.

\section{Références}

Bossé-Andrieu, Jacqueline, Cajolet-Laganière, Hélène et Pamela Russell (1994). «La rédaction professionnelle en français au Canada: résultats d'une enquête». Document manuscrit paru ultérieurement dans Journal of Technical Writing and Communication 24, 3 (1994). [Ce document ayant été porté à notre connaissance une fois notre étude terminée, nous n'avons pas pu tenir compte de ses conclusions. Nous tenions, toutefois, à le citer.]

Boutry, Pascale et Lyse Contant (1991). "Contrôler la qualité, ce n'est pas «nécessairement» révisen». Circuit (décembre 1991): 4-5.

Cornibert, Geneviève (1991). «Lettre ouverte aux gestionnaires de services linguistiques: une question de survie». Circuit (décembre 1991): 3-4.

Larivière, Louise (1986). «La rédaction dans l'entreprise: technique ou professionnelle?». Technostyle 5, 2 (été 1986): 1-10.

Verrier, Danielle (1991). «Gestionnaires de services linguistiques: à vos pupitres!». Circuit (décembre 1991): 3. 